\title{
Degrees of Freedom for the 4 User SIMO Interference Channel
}

\author{
Tiangao Gou, Syed A. Jafar \\ Electrical Engineering and Computer Science \\ University of California Irvine, Irvine, California, 92697, USA \\ Email: \{tgou,syed\}@uci.edu
}

\begin{abstract}
The 4 user single input multiple output (SIMO) Gaussian interference channel where each transmitter has a single antenna and each receiver has two antennas is studied. We show that if the channel coefficients are time-varying and drawn from a continuous distribution, the maximum number of spatial degrees of freedom per orthogonal time dimension for this channel is $\frac{8}{3}$ almost surely. Achievability is based on the idea of interference alignment, i.e., signal spaces are aligned at receiver where they constitute interference while they are separable at receivers where they are desired.
\end{abstract}

\section{INTRODUCTION}

Interference management is one challenging problem in wireless system design. Multiple input multiple output (MIMO) communication technique provides an important way to communicate over wireless channels. The capacity region of the Gaussian interference channel with multiple antenna nodes is studied in [1]-[3]. The MIMO interference channel is also studied from a degrees of freedom perspective ${ }^{1}$ [4], [5].

In [3], the authors study the achievable rate region of the multiple input single output (MISO) interference channel by treating interference as noise for arbitrary SNR. They parameterize the Pareto boundary of the MISO Gaussian interference channel for arbitrary number of users and antennas at the transmitter as long as the number of antennas is larger than that of users. For the 2 user case, they show that the optimal beamforming directions are a linear combination of maximum ratio transmission vectors and the zero forcing vectors. However, for the case when the number of antennas is less than that of users, it is still not known what the optimal beamforming directions are. Intuitively, this is because when the number of antennas is less than that of users, it is impossible for each user to choose beamforming vectors to ensure no interference is created at all other users. The same problem happens when we study this channel from a degrees of freedom perspective. For the 2 user MISO interference channel with 2 transmit antennas and a single receive antenna, it is easy to see 2 degrees of freedom can be achieved if each user chooses zero forcing beamforming vector so that no interference is created at another user. In fact, this is also the total number of degrees of freedom of this channel. However, for the 3 user MISO interference channel, it is impossible for each user to choose beamforming vectors so that no interference is created at all other users. As a result,

\footnotetext{
${ }^{1}$ If the sum capacity can be expressed as $C_{\Sigma}(S N R)=\eta \log (S N R)+$ $o(\log (S N R))$ then we say that the channel has $\eta$ degrees of freedom.
}

only 2 degrees of freedom can be achieved by zero forcing. Can we do better than merely zero forcing? What is the total number of degrees of freedom of the 3 user or even more user MISO interference channel with 2 transmit antennas anyway?

In this work we focus on the 4 user SIMO interference channel where each transmitter with a single antenna has a message for its corresponding receiver with two antennas. We provide achievability as well as converse arguments for the maximum degrees of freedom for this channel if the channel coefficients are time-varying and drawn from a continuous distribution. Since the innerbound and outerbound are tight, the precise number of degrees of freedom is $\frac{8}{3}$ per orthogonal time dimension. The constructive proof of achievability of the degrees of freedom is based on the idea of interference alignment. However, the interference alignment scheme proposed in this paper is different from all prior schemes.

All prior interference alignment schemes [6] (including the ones for the $X$ channel [7]) explicitly achieve one-to-one alignment of signal vectors, i.e., to minimize the dimension of space spanned by interference signal vectors, one signal vector from an interferer and one signal vector from another interferer are aligned along the same line at the desired receivers. For example, consider 3 user SISO interference channel with 2 symbol extension or 3 user MIMO interference channel where each node has 2 antennas. We need to choose beamforming vectors $\mathbf{v}^{[2]}$ and $\mathbf{v}^{[3]}$ at transmitter 2 and 3 , respectively so that they cast overlapping shadow at receiver 1 , i.e.,

$$
\mathbf{H}^{[12]} \mathbf{V}^{[2]}=\mathbf{H}^{[13]} \mathbf{V}^{[3]}
$$

where $\mathbf{H}^{[12]}$ and $\mathbf{H}^{[13]}$ are $2 \times 2$ channel matrices from transmitter 2 and 3 to receiver 1, respectively. However, such an alignment is not feasible on the SIMO channel. Notice that the solution to the condition mentioned above exists only when the range of the two channel matrices has intersection. The channel matrix for the 2 symbol extension SIMO channel is $4 \times 2$. The range of such two channel matrices has intersection with probability zero if the channel coefficients are drawn from a continuous distribution. Thus, this scheme does not work for SIMO channel. In this work, we seek other interference alignment schemes for the SIMO channel. We note that the results of this paper can be generalized to the $K$ user SIMO Gaussian interference channel with arbitrary number of antennas at receiver. Interested reader can refer to the journal version of this paper. 


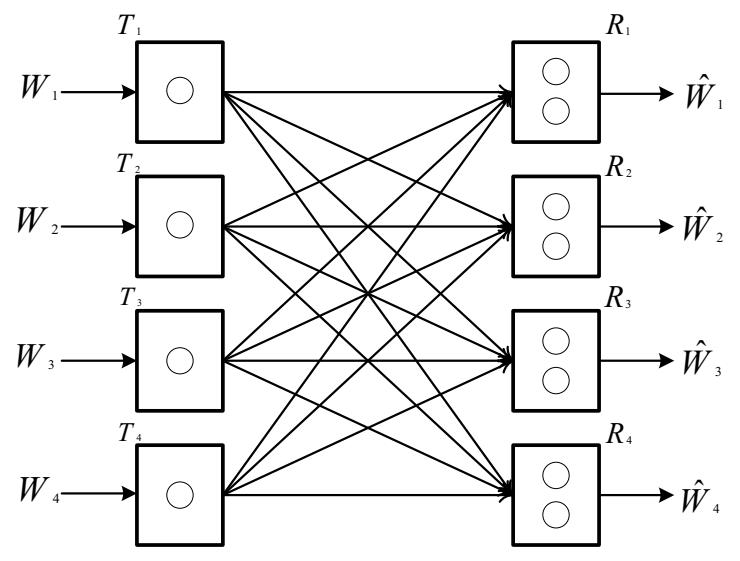

Fig. 1. The 4 user SIMO interference channel

Remark: If interference alignment is achieved on the SIMO channel it can also be achieved on the MISO channel, due to a reciprocity of alignment [8]. Thus, our results also establish the degrees of freedom for the 4 user MISO interference channel, i.e., the maximum degrees of freedom of the 4 user MISO interference channel where each transmitter has 2 antennas is $\frac{8}{3}$.

\section{SySTEM MOdeL}

As shown in Figure 1, the 4 user SIMO interference channel is comprised of 4 transmitters and 4 receivers. Each transmitter has a single antenna and each receiver has 2 antennas. The channel output at the $k^{t h}$ receiver over the $t^{t h}$ time slot is characterized by the following input output relationships:

$$
\mathbf{Y}^{[k]}(t)=\sum_{j=1}^{4} \mathbf{h}^{[k j]}(t) x^{[j]}(t)+\mathbf{N}^{[k]}(t)
$$

where, $k \in\{1,2,3,4\}$ is the user index, $t \in \mathbb{N}$ is the time slot index, $\mathbf{Y}^{[k]}(t)$ is the output signal of the $k^{t h}$ receiver, $x^{[j]}(t)$ is the input signal of the $j^{t h}$ transmitter, $\mathbf{h}^{[k j]}(t)$ is the $2 \times 1$ channel vector from transmitter $j$ to receiver $k$ over the $t^{t h}$ time slot and $\mathbf{N}^{[k]}(t)$ is the $2 \times 1$ additive white Gaussian noise (AWGN) vector at the $k^{t h}$ receiver.

We assume that all channel coefficient values are drawn i.i.d. from a continuous distribution and the absolute value of all the channel coefficients is bounded between a non-zero minimum value and a finite maximum value. The channel coefficient values vary at every channel use. Perfect knowledge of all channel coefficients is available to all transmitters and receivers.

As shown in Figure 1, transmitters 1,2,3,4 have independent messages $W_{1}, W_{2}, W_{3}, W_{4}$ intended for receivers $1,2,3,4$, respectively. The total power across all transmitters is assumed to be equal to $\rho$. We indicate the size of the message set by $\left|W_{i}(\rho)\right|$. For codewords spanning $t_{0}$ channel uses, the rates $R_{i}(\rho)=\frac{\log \left|W_{i}(\rho)\right|}{t_{0}}$ are achievable if the probability of error for all messages can be simultaneously made arbitrarily small by choosing an appropriately large $t_{0}$.
The capacity region $\mathcal{C}(\rho)$ of the 4 user SIMO interference channel is the set of all achievable rate tuples $\mathbf{R}(\rho)=$ $\left(R_{1}(\rho), R_{2}(\rho), R_{3}(\rho), R_{4}(\rho)\right)$.

\section{A. Degrees of Freedom}

We define the spatial degrees of freedom as:

$$
\eta \triangleq \lim _{\rho \rightarrow \infty} \frac{C_{\Sigma}(\rho)}{\log (\rho)}
$$

where $C_{\Sigma}(\rho)$ is the sum capacity at $\operatorname{SNR} \rho$.

\section{B. The M Symbol Extension Channel Model}

We collectively denote the $M$ transmitted symbols over $M$ time slots as a supersymbol. We call this $M$ symbol extension of the channel. For example, consider a point to point communication system with a single transmit antenna and 2 receive antennas. After 2 channel uses, the input output equation for each orthogonal time can be collectively written in the vector form as a MIMO channel with 2 antennas at the transmitter and 4 antennas at the receiver:

$$
\left[\begin{array}{l}
\mathbf{Y}(1) \\
\mathbf{Y}(2)
\end{array}\right]=\left[\begin{array}{ll}
\mathbf{h}(1) & \mathbf{0}_{2 \times 1} \\
\mathbf{0}_{2 \times 1} & \mathbf{h}(2)
\end{array}\right]\left[\begin{array}{l}
x(1) \\
x(2)
\end{array}\right]+\left[\begin{array}{l}
\mathbf{N}(1) \\
\mathbf{N}(2)
\end{array}\right]
$$

where over the $k^{t h}$ orthogonal time slot, $\mathbf{Y}(k), \mathbf{h}(k), x(k)$, $\mathbf{N}(k)$ represent the output of the receiver, the channel vector, the input signal and the noise vector at the receiver, respectively. This equation can be also expressed by substituting the vector into its entries as

$$
\left[\begin{array}{l}
y_{1}(1) \\
y_{2}(1) \\
y_{1}(2) \\
y_{2}(2)
\end{array}\right]=\left[\begin{array}{cc}
h_{1}(1) & 0 \\
h_{2}(1) & 0 \\
0 & h_{1}(2) \\
0 & h_{2}(2)
\end{array}\right]\left[\begin{array}{l}
x(1) \\
x(2)
\end{array}\right]+\left[\begin{array}{c}
n_{1}(1) \\
n_{2}(1) \\
n_{1}(2) \\
\left.n_{2}(2)\right)
\end{array}\right]
$$

We can exchange the order of the second and third row of the received vector. The corresponding input output relation is

$$
\underbrace{\left[\begin{array}{l}
y_{1}(1) \\
y_{1}(2) \\
y_{2}(1) \\
y_{2}(2)
\end{array}\right]}_{\overline{\mathbf{Y}}}=\underbrace{\left[\begin{array}{cc}
h_{1}(1) & 0 \\
0 & h_{1}(2) \\
h_{2}(1) & 0 \\
0 & h_{2}(2)
\end{array}\right]}_{\overline{\mathbf{H}}} \underbrace{\left[\begin{array}{c}
x(1) \\
x(2)
\end{array}\right]}_{\overline{\mathbf{X}}}+\underbrace{\left[\begin{array}{l}
n_{1}(1) \\
n_{1}(2) \\
n_{2}(1) \\
n_{2}(2)
\end{array}\right]}_{\overline{\mathbf{N}}}
$$

Now consider the $M$ symbol extension channel. The input output relationship can be expressed as

$$
\overline{\mathbf{Y}}(t)=\overline{\mathbf{H}}(t) \overline{\mathbf{X}}(t)+\overline{\mathbf{N}}(t)
$$

where $\overline{\mathbf{X}}$ is an $M \times 1$ column vector representing the $M$ symbol extension of the transmitted symbol $x$, i.e.,

$$
\overline{\mathbf{X}}(t) \triangleq\left[\begin{array}{c}
x(M(t-1)+1) \\
x(M(t-1)+2) \\
\vdots \\
x(M t)
\end{array}\right]
$$

Similarly, $\overline{\mathbf{Y}}$ and $\overline{\mathbf{N}}$ represent $M$ symbol extensions of the $\mathbf{Y}$ and $\mathbf{N}$, respectively. $\overline{\mathbf{H}}$ is a $2 M \times M$ matrix representing the 
$M$ symbol extension of the channel, i.e.,

$$
\overline{\mathbf{H}} \triangleq\left[\begin{array}{cccc}
h_{1}(M(t-1)+1) & 0 & \cdots & 0 \\
0 & h_{1}(M(t-1)+2) & \cdots & 0 \\
\vdots & \ldots & \ddots & \vdots \\
0 & 0 & \cdots & h_{1}(M t) \\
h_{2}(M(t-1)+1) & 0 & \cdots & 0 \\
0 & h_{2}(M(t-1)+2) & \cdots & \vdots \\
\vdots & \cdots & \ddots & \vdots \\
0 & 0 & \cdots & h_{2}(M t)
\end{array}\right]
$$

\section{DEGREES OF FREEDOM FOR THE 4 USER SIMO INTERFERENCE CHANNEL}

We present the main results of this paper in the following theorem.

Theorem 1: For the 4 user SIMO Gaussian interference channel with a single antenna at each transmitter and 2 antennas at each receiver, the total number of degrees of freedom per orthogonal time dimension is equal to $\frac{8}{3}$, i.e.,

$$
\eta=d_{1}+d_{2}+d_{3}+d_{4}=\frac{8}{3}
$$

where $d_{i}$ represents the individual degrees of freedom achieved by user $i$. In the following section, we will show that both the innerbound (achievability) and outerbound (converse) on the degrees of freedom are equal to $\frac{8}{3}$. Since the innerbound and outerbound match, theorem 1 is proved.

\section{A. Converse for Theorem 1}

Proof: In [4], it is shown that the degrees of freedom for a two user MIMO Gaussian interference channel with $M_{1}, M_{2}$ antennas at transmitter 1,2 and $N_{1}, N_{2}$ antennas at the corresponding receivers is $\min \left\{M_{1}+M_{2}, N_{1}+N_{2}\right.$, $\left.\max \left(M_{1}, N_{2}\right), \max \left(M_{2}, N_{1}\right)\right\}$. From this result, the degrees of freedom for two user SIMO interference channel with 2 antennas at all receiver nodes is 2 . For the 3 user case, if we allow full cooperation between the first two transmitters and full cooperation between their corresponding receivers, then it is equivalent to a two user MIMO interference channel where the transmitter has 2 antennas and 1 antenna, respectively and their corresponding receiver has 4 antennas and 2 antennas respectively. The degrees of freedom for this channel is also 2. Since allowing transmitters and receivers to cooperate does not hurt the capacity, the degrees of freedom of the original 3 user interference channel is no more than 2. For the 4 user case, picking any 3 users among 4 users gives an outerbound:

$$
d_{i}+d_{j}+d_{k} \leq 2 \quad \forall i, j, k \in\{1,2,3,4\}, \quad i \neq j \neq k
$$

Adding up all such inequalities, we get the outerbound of the 4 user SIMO interference channel:

$$
d_{1}+d_{2}+d_{3}+d_{4} \leq \frac{8}{3}
$$

\section{B. Achievability Proof for Theorem 1}

In this section, we provide an achievable scheme to show that for the 4 user SIMO interference channel, user 1, 2, 3, 4 can achieve $2(n+1)^{8}, 2(n+1)^{8}, 2(n+1)^{8}, 2 n^{8}$ degrees of freedom respectively for any $n \in \mathbb{N}$ on the $M=3(n+1)^{8}$ symbol extension channel. Therefore, user $i$ can achieve $d_{i}$ degrees of freedom per channel use, where

$$
d_{i}=\frac{2(n+1)^{8}}{3(n+1)^{8}} \quad i=1,2,3 \quad d_{4}=\frac{2 n^{8}}{3(n+1)^{8}}
$$

This implies that

$$
d_{1}+d_{2}+d_{3}+d_{4} \geq \sup _{n} \frac{3 \times 2(n+1)^{8}+2 n^{8}}{3(n+1)^{8}}=\frac{8}{3}
$$

On the extended channel, the signal vector at the $k^{t h}$ user's receiver can be expressed as:

$$
\overline{\mathbf{Y}}^{[k]}(t)=\sum_{j=1}^{4} \overline{\mathbf{H}}^{[k j]} \overline{\mathbf{X}}^{[j]}(t)+\overline{\mathbf{N}}^{[k]}(t)
$$

where $\overline{\mathbf{X}}^{[j]}$ is an $M \times 1$ column vector representing the $M$ symbol extension of the transmitted symbol $x^{[j]}$. Similarly, $\overline{\mathbf{Y}}$ and $\overline{\mathbf{N}}$ represent $M$ symbol extensions of the $\mathbf{Y}$ and $\mathbf{N}$ respectively. $\overline{\mathbf{H}}$ is a $2 M \times M$ matrix representing the $M$ symbol extension of the channel, i.e.,

$$
\overline{\mathbf{H}}^{[k j]} \triangleq\left[\begin{array}{c}
\overline{\mathbf{H}}_{1}^{[k j]} \\
\overline{\mathbf{H}}_{2}^{[k j]}
\end{array}\right]
$$

where

$\overline{\mathbf{H}}_{i}^{[k j]}=\left[\begin{array}{cccc}h_{i}^{[k j]}(M(t-1)+1) & 0 & \cdots & 0 \\ 0 & h_{i}^{[k j]}(M(t-1)+2) & \cdots & 0 \\ \vdots & \cdots & \ddots & \vdots \\ 0 & 0 & \cdots & h_{i}^{[k j]}(M t)\end{array}\right]$

where $i=1,2$. Message $W_{j}(j=1,2,3)$ is encoded at transmitter $j$ into $2(n+1)^{8}$ independent streams $x_{m}^{[j]}, m=$ $1,2, \ldots, 2(n+1)^{8}$ along vectors $\mathbf{v}_{m}^{[1]}$ so that $\overline{\mathbf{X}}^{[j]}(t)$ is

$$
\overline{\mathbf{X}}^{[j]}(t)=\sum_{m=1}^{2(n+1)^{8}} x_{m}^{[j]}(t) \mathbf{v}_{m}^{[1]}=\overline{\mathbf{V}}^{[1]} \mathbf{X}^{[j]}(t), \quad j=1,2,3
$$

where $\mathbf{X}^{[j]}(t)$ is a $2(n+1)^{8} \times 1$ column vector and $\overline{\mathbf{V}}^{[1]}$ is a $3(n+1)^{8} \times 2(n+1)^{8}$ dimensional matrix. Note that transmitter 1,2,3 send their respective data streams along the same beamforming vectors. $W_{4}$ is encoded at transmitter 4 into $2 n^{8}$ independent streams $x_{m}^{[4]}, m=1,2, \ldots, 2 n^{8}$ along vectors $\mathbf{v}_{m}^{[4]}$ as

$$
\overline{\mathbf{X}}^{[4]}(t)=\sum_{m=1}^{2 n^{8}} x_{m}^{[4]}(t) \mathbf{v}_{m}^{[4]}=\overline{\mathbf{V}}^{[4]} \mathbf{X}^{[4]}(t)
$$

The received signal at the $k^{t h}$ receiver can then be written as $\overline{\mathbf{Y}}^{[k]}(t)=\sum_{j=1}^{3} \overline{\mathbf{H}}^{[k j]} \overline{\mathbf{V}}^{[1]} \mathbf{X}^{[j]}(t)+\overline{\mathbf{H}}^{[k 4]} \overline{\mathbf{V}}^{[4]} \mathbf{X}^{[4]}(t)+\overline{\mathbf{N}}^{[k]}(t)$

Note that $\overline{\mathbf{Y}}^{[k]}(t)$ is a $2 M=6(n+1)^{8} \times 1$ vector which provides a $6(n+1)^{8}$ dimensional signal space at each receiver. We wish to divide this received signal space into two disjoint subspaces, i.e., the intersection of the two subspaces is $\{0\}$. One such subspace is spanned by the desired signal vectors, and another is spanned by the interference signal vectors. As a 
result, each receiver can get interference free signals by zeroforcing the signals in the subspace spanned by the interference signals. To achieve this, we should carefully design columns of $\overline{\mathbf{V}}^{[1]}$ and $\overline{\mathbf{V}}^{[4]}$ in such a manner that simultaneously at each receiver the space spanned by interference signal vectors is disjoint with the space spanned by the desired signal vectors. In other words, we align the interference into the space which is disjoint with that spanned by the desired signal. We call this interference alignment.

At receiver 1 , the desired signal occupies $2(n+1)^{8}$ dimensional space spanned by columns of $\overline{\mathbf{H}}^{[11]} \overline{\mathbf{V}}^{[1]}$. The interference signal vectors are columns of matrix $\left[\overline{\mathbf{H}}^{[12]} \overline{\mathbf{V}}^{[1]} \quad \overline{\mathbf{H}}^{[13]} \overline{\mathbf{V}}^{[1]} \quad \overline{\mathbf{H}}^{[14]} \overline{\mathbf{V}}^{[4]}\right]$. In general, these interference signal vectors span a $4(n+1)^{8}+2 n^{8}$ dimensional subspace in the $6(n+1)^{8}$ dimensional signal space. To decode the desired signal by zero-forcing the interference signals, we wish to choose $\overline{\mathbf{V}}^{[1]}$ and $\overline{\mathbf{V}}^{[4]}$ so that the following two conditions are satisfied:

(1) The dimension of the space spanned by interference signal vectors can not be more than $4(n+1)^{8}$.

(2) The $2(n+1)^{8}$ dimensional space spanned by the desired signal vectors does not have intersection with signal space spanned by the interference signal vectors.

Mathematically, the first condition can be expressed as

$$
\operatorname{rank}\left(\left[\overline{\mathbf{H}}^{[12]} \overline{\mathbf{V}}^{[1]} \quad \overline{\mathbf{H}}^{[13]} \overline{\mathbf{V}}^{[1]} \quad \overline{\mathbf{H}}^{[14]} \overline{\mathbf{V}}^{[4]}\right]\right) \leq 4(n+1)^{8}
$$

Notice that all the interference vectors from transmitter 2,3 span a $4(n+1)^{8}$ dimensional subspace. Hence, we can align the interference signal vectors from transmitter 4 within this $4(n+1)^{8}$ dimensional subspace. This can be achieved if any column of $\overline{\mathbf{H}}^{[14]} \overline{\mathbf{V}}^{[4]}$ is a linear combination of column vectors of $\overline{\mathbf{H}}^{[12]} \overline{\mathbf{V}}^{[1]}$ and column vectors of $\overline{\mathbf{H}}^{[13]} \overline{\mathbf{V}}^{[1]}$, i.e., $\forall m \in$ $\left\{1,2, \ldots, 2 n^{8}\right\}, \exists k_{m}, j_{m} \in\left\{1,2, \ldots, 2(n+1)^{8}\right\}$ so that

$$
\begin{gathered}
\overline{\mathbf{H}}^{[12]} \mathbf{v}_{k_{m}}^{[1]}+\overline{\mathbf{H}}^{[13]} \mathbf{v}_{j_{m}}^{[1]}=\overline{\mathbf{H}}^{[14]} \mathbf{v}_{m}^{[4]} \\
\Rightarrow\left[\begin{array}{c}
\overline{\mathbf{H}}_{1}^{[12]} \\
\overline{\mathbf{H}}_{2}^{[12]}
\end{array}\right] \mathbf{v}_{k_{m}}^{[1]}+\left[\begin{array}{c}
\overline{\mathbf{H}}_{1}^{[13]} \\
\overline{\mathbf{H}}_{2}^{[13]}
\end{array}\right] \mathbf{v}_{j_{m}}^{[1]}=\left[\begin{array}{c}
\overline{\mathbf{H}}_{1}^{[14]} \\
\overline{\mathbf{H}}_{2}^{[14]}
\end{array}\right] \mathbf{v}_{m}^{[4]} \\
\Rightarrow\left\{\begin{array}{l}
\overline{\mathbf{H}}_{1}^{[12]} \mathbf{v}_{k_{m}}^{[1]}+\overline{\mathbf{H}}_{1}^{[13]} \mathbf{v}_{j_{m}}^{[1]}=\overline{\mathbf{H}}_{1}^{[14]} \mathbf{v}_{m}^{[4]} \\
\overline{\mathbf{H}}_{2}^{[12]} \mathbf{v}_{k_{m}}^{[1]}+\overline{\mathbf{H}}_{2}^{[13]} \mathbf{v}_{j_{m}}^{1]}=\overline{\mathbf{H}}_{2}^{[14]} \mathbf{v}_{m}^{[4]}
\end{array}\right.
\end{gathered}
$$

Recall that $\overline{\mathbf{H}}_{i}^{[k j]}$ is a $3(n+1)^{8} \times 3(n+1)^{8}$ diagonal matrix whose entries are drawn i.i.d from a continuous distribution. Thus it is full rank with probability one. Then the above equation can be simplified as

$$
\left\{\begin{array}{l}
\mathbf{v}_{k_{m}}^{[1]}=\mathbf{A}_{1}^{-1} \mathbf{B}_{1} \mathbf{v}_{m}^{[4]} \\
\mathbf{v}_{j_{m}}^{[1]}=\mathbf{A}_{2}^{-1} \mathbf{B}_{2} \mathbf{v}_{m}^{[4]}
\end{array}\right.
$$

where

$$
\begin{aligned}
& \mathbf{A}_{1}=\left(\overline{\mathbf{H}}_{1}^{[13]}\right)^{-1} \overline{\mathbf{H}}_{1}^{[12]}-\left(\overline{\mathbf{H}}_{2}^{[13]}\right)^{-1} \overline{\mathbf{H}}_{2}^{[12]} \\
& \mathbf{B}_{1}=\left(\overline{\mathbf{H}}_{1}^{[13]}\right)^{-1} \overline{\mathbf{H}}_{1}^{[14]}-\left(\overline{\mathbf{H}}_{2}^{[13]}\right)^{-1} \overline{\mathbf{H}}_{2}^{[14]}
\end{aligned}
$$

$$
\begin{aligned}
& \mathbf{A}_{2}=\left(\overline{\mathbf{H}}_{1}^{[12]}\right)^{-1} \overline{\mathbf{H}}_{1}^{[13]}-\left(\overline{\mathbf{H}}_{2}^{[12]}\right)^{-1} \overline{\mathbf{H}}_{2}^{[13]} \\
& \mathbf{B}_{2}=\left(\overline{\mathbf{H}}_{1}^{[12]}\right)^{-1} \overline{\mathbf{H}}_{1}^{[14]}-\left(\overline{\mathbf{H}}_{2}^{[12]}\right)^{-1} \overline{\mathbf{H}}_{2}^{[14]}
\end{aligned}
$$

Furthermore, this condition can be expressed as

$$
\left\{\begin{array}{rll}
\mathbf{A}_{1}^{-1} \mathbf{B}_{1} \overline{\mathbf{V}}^{[4]} & \prec \overline{\mathbf{V}}^{[1]} \\
\mathbf{A}_{2}^{-1} \mathbf{B}_{2} \overline{\mathbf{V}}^{[4]} & \prec \overline{\mathbf{V}}^{[1]}
\end{array}\right.
$$

where $\mathbf{P} \prec \mathbf{Q}$ means that the set of column vectors of matrix $\mathbf{P}$ is a subset of the set of column vectors of matrix $\mathbf{Q}$.

At the same time, to extract $2(n+1)^{8}$ interference free signal at receiver 2,3 we need:

$$
\begin{array}{r}
\left\{\begin{array}{rll}
\mathbf{A}_{3}^{-1} \mathbf{B}_{3} \overline{\mathbf{V}}^{[4]} & \prec & \overline{\mathbf{V}}^{[1]} \\
\mathbf{A}_{4}^{-1} \mathbf{B}_{4} \overline{\mathbf{V}}^{[4]} & \prec & \overline{\mathbf{V}}^{[1]}
\end{array}\right. \\
\left\{\begin{array}{lll}
\mathbf{A}_{5}^{-1} \mathbf{B}_{5} \overline{\mathbf{V}}^{[4]} & \prec & \overline{\mathbf{V}}^{[1]} \\
\mathbf{A}_{6}^{-1} \mathbf{B}_{6} \overline{\mathbf{V}}^{[4]} & \prec & \overline{\mathbf{V}}^{[1]}
\end{array}\right.
\end{array}
$$

where

$$
\begin{aligned}
& \mathbf{A}_{3}=\left(\overline{\mathbf{H}}_{1}^{[23]}\right)^{-1} \overline{\mathbf{H}}_{1}^{[21]}-\left(\overline{\mathbf{H}}_{2}^{[23]}\right)^{-1} \overline{\mathbf{H}}_{2}^{[21]} \\
& \mathbf{B}_{3}=\left(\overline{\mathbf{H}}_{1}^{[23]}\right)^{-1} \overline{\mathbf{H}}_{1}^{[24]}-\left(\overline{\mathbf{H}}_{2}^{[23]}\right)^{-1} \overline{\mathbf{H}}_{2}^{[24]} \\
& \mathbf{A}_{4}=\left(\overline{\mathbf{H}}_{1}^{[21]}\right)^{-1} \overline{\mathbf{H}}_{1}^{[23]}-\left(\overline{\mathbf{H}}_{2}^{[21]}\right)^{-1} \overline{\mathbf{H}}_{2}^{[23]} \\
& \mathbf{B}_{4}=\left(\overline{\mathbf{H}}_{1}^{[21]}\right)^{-1} \overline{\mathbf{H}}_{1}^{[24]}-\left(\overline{\mathbf{H}}_{2}^{[21]}\right)^{-1} \overline{\mathbf{H}}_{2}^{[24]} \\
& \mathbf{A}_{5}=\left(\overline{\mathbf{H}}_{1}^{[32]}\right)^{-1} \overline{\mathbf{H}}_{1}^{[31]}-\left(\overline{\mathbf{H}}_{2}^{[32]}\right)^{-1} \overline{\mathbf{H}}_{2}^{[31]} \\
& \mathbf{B}_{5}=\left(\overline{\mathbf{H}}_{1}^{[32]}\right)^{-1} \overline{\mathbf{H}}_{1}^{[34]}-\left(\overline{\mathbf{H}}_{2}^{[32]}\right)^{-1} \overline{\mathbf{H}}_{2}^{[34]} \\
& \mathbf{A}_{6}=\left(\overline{\mathbf{H}}_{1}^{[31]}\right)^{-1} \overline{\mathbf{H}}_{1}^{[32]}-\left(\overline{\mathbf{H}}_{2}^{[31]}\right)^{-1} \overline{\mathbf{H}}_{2}^{[32]} \\
& \mathbf{B}_{6}=\left(\overline{\mathbf{H}}_{1}^{[31]}\right)^{-1} \overline{\mathbf{H}}_{1}^{[34]}-\left(\overline{\mathbf{H}}_{2}^{[31]}\right)^{-1} \overline{\mathbf{H}}_{2}^{[34]}
\end{aligned}
$$

At receiver 4 , the desired signal needs $2 n^{8}$ dimensional interference free space. Thus, the dimension of space spanned by interference signal vectors should be no more than $6(n+$ $1)^{8}-2 n^{8}$. Mathematically, we need

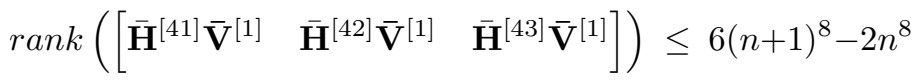

This can be achieved if there are $2 n^{8}$ columns of $\overline{\mathbf{H}}^{[42]} \overline{\mathbf{V}}^{[1]}$ each of which is a linear combination of column vectors of $\overline{\mathbf{H}}^{[41]} \overline{\mathbf{V}}^{[1]}$ and column vectors of $\overline{\mathbf{H}}^{[43]} \overline{\mathbf{V}}^{[1]}$. From equation (2), we have

$$
\mathbf{v}_{k_{m}}^{[1]}=\mathbf{A}_{1}^{-1} \mathbf{B}_{1} \mathbf{v}_{m}^{[4]} \quad m=1,2, \ldots, 2 n^{8}
$$


Without loss of generosity, we let these $2 n^{8}$ vectors to satisfy the condition mentioned above, i.e.,

$$
\begin{array}{r}
\forall \mathbf{v}_{k_{m}}^{[1]}, m=1,2, \ldots, 2 n^{8}, \exists l_{m}, p_{m} \in\left\{1,2, \ldots, 2(n+1)^{8}\right\} \\
k_{m} \neq l_{m} \neq p_{m},
\end{array}
$$

so that

$$
\overline{\mathbf{H}}^{[41]} \mathbf{v}_{l_{m}}^{[1]}+\overline{\mathbf{H}}^{[43]} \mathbf{v}_{p_{m}}^{[1]}=\overline{\mathbf{H}}^{[42]} \mathbf{v}_{k_{m}}^{[1]}=\overline{\mathbf{H}}^{[42]} \mathbf{A}_{1}^{-1} \mathbf{B}_{1} \mathbf{v}_{m}^{[4]}
$$

By the similar procedure used at receiver 1, this equation can be simplified as

$$
\left\{\begin{array}{lll}
\mathbf{A}_{7}^{-1} \mathbf{B}_{7} \overline{\mathbf{V}}^{[4]} & \prec \overline{\mathbf{V}}^{[1]} \\
\mathbf{A}_{8}^{-1} \mathbf{B}_{8} \overline{\mathbf{V}}^{[4]} & \prec \overline{\mathbf{V}}^{[1]}
\end{array}\right.
$$

where

$$
\begin{gathered}
\mathbf{A}_{7}=\left(\overline{\mathbf{H}}_{1}^{[43]}\right)^{-1} \overline{\mathbf{H}}_{1}^{[41]}-\left(\overline{\mathbf{H}}_{2}^{[43]}\right)^{-1} \overline{\mathbf{H}}_{2}^{[41]} \\
\mathbf{A}_{8}=\left(\overline{\mathbf{H}}_{1}^{[41]}\right)^{-1} \overline{\mathbf{H}}_{1}^{[43]}-\left(\overline{\mathbf{H}}_{2}^{[41]}\right)^{-1} \overline{\mathbf{H}}_{2}^{[43]} \\
\mathbf{B}_{7}=\left(\overline{\mathbf{H}}_{1}^{[43]}\right)^{-1} \overline{\mathbf{H}}_{1}^{[42]} \mathbf{A}_{1}^{-1} \mathbf{B}_{1}-\left(\overline{\mathbf{H}}_{2}^{[43]}\right)^{-1} \overline{\mathbf{H}}_{1}^{[42]} \mathbf{A}_{1}^{-1} \mathbf{B}_{1} \\
\mathbf{B}_{8}=\left(\overline{\mathbf{H}}_{1}^{[41]}\right)^{-1} \overline{\mathbf{H}}_{1}^{[42]} \mathbf{A}_{1}^{-1} \mathbf{B}_{1}-\left(\overline{\mathbf{H}}_{2}^{[41]}\right)^{-1} \overline{\mathbf{H}}_{2}^{[42]} \mathbf{A}_{1}^{-1} \mathbf{B}_{1}
\end{gathered}
$$

Therefore, to get a small enough interference space, we should choose $\overline{\mathbf{V}}^{[1]}$ and $\overline{\mathbf{V}}^{[4]}$ to satisfy:

$$
\mathbf{T}_{i} \overline{\mathbf{V}}^{[4]} \prec \overline{\mathbf{V}}^{[1]}
$$

where $\mathbf{T}_{i}=\mathbf{A}_{i}^{-1} \mathbf{B}_{i}, \quad i=1, \ldots, 8$ and $\mathbf{T}_{i}$ are diagonal matrices with non-zero entries.

Let $\mathbf{w}$ be a $3(n+1)^{8} \times 1$ column vector $\mathbf{w}=\left[\begin{array}{llll}1 & 1 & \ldots & 1\end{array}\right]^{T}$. We need to choose $2 n^{8}$ column vectors for $\overline{\mathbf{V}}^{[4]}$ and $2(n+1)^{8}$ column vectors for $\overline{\mathbf{V}}^{[1]}$. The sets of column vectors of $\overline{\mathbf{V}}^{[4]}$ and $\overline{\mathbf{V}}^{[1]}$ are chosen to be equal to the sets $\bar{V}^{[4]}$ and $\bar{V}^{[1]}$ where

$$
\begin{aligned}
\bar{V}^{[4]}= & \left\{\left(\prod_{i=1}^{8} \mathbf{T}_{i}^{\alpha_{i}}\right) \mathbf{w}:\left(\alpha_{1}, \ldots, \alpha_{8}\right) \in\{1, \ldots, n\}\right\} \quad \cup \\
& \left\{\left(\prod_{i=1}^{8} \mathbf{T}_{i}^{\beta_{i}}\right) \mathbf{w}:\left(\beta_{1}, \ldots, \beta_{8}\right) \in\{n+2, \ldots, 2 n+1\}\right\} \\
\bar{V}^{[1]}= & \left\{\left(\prod_{i=1}^{8} \mathbf{T}_{i}^{\alpha_{i}}\right) \mathbf{w}:\left(\alpha_{1}, \ldots, \alpha_{8}\right) \in\{1, \ldots, n+1\}\right\} \quad \cup \\
& \left\{\left(\prod_{i=1}^{8} \mathbf{T}_{i}^{\beta_{i}}\right) \mathbf{w}:\left(\beta_{1}, \ldots, \beta_{8}\right) \in\{n+2, \ldots, 2 n+2\}\right\}
\end{aligned}
$$

For example, when $n=1$, the set $\bar{V}^{[4]}$ consists of two elements, i.e., $\bar{V}^{[4]}=\left\{\left(\prod_{i=1}^{8} \mathbf{T}_{i}\right) \mathbf{w} \quad\left(\prod_{i=1}^{8} \mathbf{T}_{i}^{3}\right) \mathbf{w}\right\}$. The set $\bar{V}^{[1]}$ consists of $2(1+1)^{8}=2^{9}$ column vectors in the form $\left\{\left(\prod_{i=1}^{8} \mathbf{T}_{i}^{\alpha_{i}}\right) \mathbf{w} \quad\left(\prod_{i=1}^{8} \mathbf{T}_{i}^{\beta_{i}}\right) \mathbf{w}\right\}$ where $\alpha_{i}(i=1,2, \ldots, 8)$ takes values 1,$2 ; \beta_{i}(i=1,2, \ldots, 8)$ takes values 3,4 . We note that all entries of $\overline{\mathbf{V}}^{[1]}$ and $\overline{\mathbf{V}}^{[4]}$ are non-zero with probability one. This can be easily seen from the fact that $\mathbf{T}_{i}$ are diagonal matrices with non-zero entries and the construction of $\overline{\mathbf{V}}^{[1]}$ and $\overline{\mathbf{V}}^{[4]}$.

Through interference alignment, we ensure that the dimension of the interference is small enough. We now need to verify that the desired signal vectors are linearly independent of the interference vectors. At receiver 1, we need to verify that

$$
\operatorname{rank}\left(\left[\overline{\mathbf{H}}^{[11]} \overline{\mathbf{V}}^{[1]} \quad \overline{\mathbf{H}}^{[12]} \overline{\mathbf{V}}^{[1]} \quad \overline{\mathbf{H}}^{[13]} \overline{\mathbf{V}}^{[1]}\right]\right)=6(n+1)^{8}
$$

Notice that $\overline{\mathbf{V}}^{[1]}$ is chosen independent of $\overline{\mathbf{H}}^{[11]}$ and $\overline{\mathbf{V}}^{[1]}$ undergoes an independent linear transformation by multiplying $\overline{\mathbf{H}}^{[11]}$. Thus, the range of $\overline{\mathbf{H}}^{[11]} \overline{\mathbf{V}}^{[1]}$ does not have intersection with the range of $\left[\overline{\mathbf{H}}^{[12]} \overline{\mathbf{V}}^{[1]} \quad \overline{\mathbf{H}}^{[13]} \overline{\mathbf{V}}^{[1]}\right]$ almost surely. Equation (3) is satisfied with probability one. For example, consider three $3 \times 3$ diagonal matrix $\mathbf{H}^{[1]}, \mathbf{H}^{[2]}, \mathbf{H}^{[3]}$ whose diagonal entries are drawn from a continuous distribution. $\mathbf{v}$ is a $3 \times 1$ vector whose entries depend on entries of $\mathbf{H}^{[2]}$, $\mathbf{H}^{[3]}$ and are non-zero with probability one. Vectors $\mathbf{H}^{[2]} \mathbf{v}$ and $\mathbf{H}^{[3]} \mathbf{v}$ span a plane in the three dimensional space. Now vector $\mathbf{v}$ undergoes a random linear transformation by multiplying $\mathbf{H}^{[1]}$. The probability that vector $\mathbf{H}^{[1]} \mathbf{v}$ lies in that plan is zero. Similar argument can be applied at receiver 2, 3, 4. As a result, each receiver can decode the signal streams by zero-forcing the interference. Therefore, $6(n+1)^{8}+2 n^{8}$ degrees of freedom are obtained on the $M=3(n+1)^{8}$ symbol extension channel which implies that $\frac{8}{3}$ degrees of freedom per orthogonal time dimension can be achieved at the original channel.

\section{CONCLusion}

We investigate the degrees of freedom for the 4 user SIMO interference channel. The motivation of this work is the potential benefits of interference alignment scheme shown recently to achieve the capacity of certain wireless networks within $o(\log (S N R))$. In this work, interference alignment scheme is also found to be optimal in achieving the degrees of freedom of the SIMO interference channel. Generalizations to general MIMO interference channel scenarios are part of ongoing work.

\section{REFERENCES}

[1] S. Vishwanath and S. Jafar, " On the Capacity of Vector Gaussian Interference Channels," IEEE ITW,2004.

[2] X. Shang, B. Chen, G. Kramer, and H. V. Poor, "On the Capacity of MIMO Interference Channels," http://arxiv.org/abs/0807.1543, 2008.

[3] E. A. Jorswieck, E. G. Larsson and D. Danev, "Complete Characterization of the Pareto Boundary for the MISO Interference Channel," in Proc. IEEE Trans. on Signal Processing, Aug. 2008.

[4] S. Jafar and M. Fakhereddin, "Degrees of freedom for the MIMO interference channel," in IEEE Transactions on Information Theory, vol. 53, pp. 2637-2642, July 2007.

[5] C. Huang, S. Jafar, "Degrees of Freedom of the MIMO Interference Channel with Cooperation and Cognition", Preprint available through http://newport.eecs.uci.edu/syed, March 2008.

[6] V. R. Cadambe and S. A. Jafar, "Interference Alignment and Degrees of Freedom of the K-User Interference Channel," IEEE Trans. on Inform. Theory, vol. 54, No.8, pp. 3425-3441, August 2008.

[7] S. Jafar and S. Shamai, "Degrees of freedom region for the mimo X channel," IEEE Trans. on Information Theory, vol. 54, pp. 151-170, Jan. 2008.

[8] K. Gomadam, V. Cadambe and S. Jafar, "Approaching the Capacity of Wireless Networks through Distributed Interference Alignment," Preprint available through http://newport.eecs.uci.edu/syed, March 2008. 